\title{
High-order maximum principles for the stability analysis of positive bilinear control systems
}

\author{
Gal Hochma ${ }^{1}$ and Michael Margaliot ${ }^{2}$
}

\begin{abstract}
We consider a continuous-time positive bilinear control system (PBCS), i.e. a bilinear control system with Metzler matrices. The positive orthant is an invariant set of such a system, and the corresponding transition matrix $C(t)$ is entrywise nonnegative for all time $t \geq 0$. Motivated by the stability analysis of positive linear switched systems (PLSSs) under arbitrary switching laws, we fix a final time $T>0$ and define a control as optimal if it maximizes the spectral radius of $C(T)$. A recent paper [2] developed a first-order necessary condition for optimality in the form of a maximum principle (MP). In this paper, we derive higher-order necessary conditions for optimality for both singular and bang-bang controls. Our approach is based on combining results on the secondorder derivative of the spectral radius of a nonnegative matrix with the generalized Legendre-Clebsch condition and the Agrachev-Gamkrelidze second-order optimality condition.
\end{abstract}

\section{Index Terms}

Positive switched systems, stability under arbitrary switching laws, variational approach, high-order maximum principles, Perron-Frobenius theory.

\section{INTRODUCTION}

Consider the continuous-time linear switched system

$$
\begin{aligned}
& \dot{x}(t)=A_{\sigma(t)} x(t), \\
& x(0)=x_{0},
\end{aligned}
$$

Research supported in part by the Israel Science Foundation (ISF).

${ }^{1} \mathrm{GH}$ is with the School of Electrical Engineering-Systems, Tel Aviv University, Israel 69978. Email: gal.hoc@gmail.com

${ }^{2} \mathrm{MM}$ (corresponding author) is with the School of Electrical Engineering-Systems and with the Sagol School of Neuroscience, Tel Aviv University, Israel 69978. Email: michaelmeeng.tau.ac.il

An abridged version of this paper was presented at the 52nd IEEE Conference on Decision and Control [1]. 
where $x: \mathbb{R}_{+} \rightarrow \mathbb{R}^{n}$ is the state vector, and $\sigma: \mathbb{R}_{+} \rightarrow\{0,1\}$ is a piecewise constant function referred to as the switching signal. This models a system that can switch between the two linear subsystems

$$
\dot{x}=A_{0} x \text { and } \dot{x}=A_{1} x
$$

Recall that (1) is said to be globally uniformly asymptotically stable (GUAS) if there exists a class $\mathcal{K} \mathcal{L}$ function 1 such that for any initial condition $x_{0} \in \mathbb{R}^{n}$ and any switching law $\sigma$, the corresponding solution of (1) satisfies

$$
|x(t)| \leq \beta\left(\left|x_{0}\right|, t\right), \text { for all } t \geq 0 .
$$

This implies in particular that

$$
\lim _{t \rightarrow \infty} x(t)=0, \quad \text { for all } \sigma \text { and all } x_{0} \in \mathbb{R}^{n},
$$

and or linear switched systems, (2) is in fact equivalent to GUAS (see, e.g., [3]). Switched systems and, in particular, their stability analysis are attracting considerable interest in the last two decades; see e.g. the survey papers [4], [5], [6], [7] and the monographs [8], [9], [10], [11], [12], [13].

It is well-known that a necessary (but not sufficient) condition for GUAS of (1) is the following.

Assumption 1: The matrix $k A_{0}+(1-k) A_{1}$ is Hurwitz for all $k \in[0,1]$.

Recall that a linear system

$$
\dot{x}=A x,
$$

with $A \in \mathbb{R}^{n \times n}$, is called positive if the positive orthant

$$
\mathbb{R}_{+}^{n}:=\left\{x \in \mathbb{R}^{n} \mid x_{i} \geq 0, i=1, \ldots, n\right\}
$$

is an invariant set of the dynamics, i.e., $x(0) \in \mathbb{R}_{+}^{n}$ implies that $x(t) \in \mathbb{R}_{+}^{n}$ for all $t \geq 0$.

Positive systems play an important role in systems and control theory because in many physical systems the state-variables represent quantities that can never attain negative values (e.g. population sizes, probabilities, concentrations, buffer loads) [14], [15], [16]. A necessary and sufficient condition for (3) to be positive is that $A$ is a Metzler matrix, that is, $a_{i j} \geq 0$ for all $i \neq j$. If $A$ is $\operatorname{Metzler}$ then $\exp (A t)$ is (entrywise) nonnegative for all $t \geq 0$. By the Perron-Frobenius theory, the spectral radius of $\exp (A t)$ (i.e.,

\footnotetext{
${ }^{1} \mathrm{~A}$ continuous function $\alpha:[0, \infty) \rightarrow[0, \infty)$ belongs to the class $\mathcal{K}$ if it is strictly increasing and $\alpha(0)=0$. A continuous function $\beta:[0, \infty) \times[0, \infty) \rightarrow[0, \infty)$ belongs to the class $\mathcal{K} \mathcal{L}$ if for each fixed $s, \beta(\cdot, s)$ belongs to $\mathcal{K}$, and for each fixed $r>0$, the mapping $\beta(r, \cdot)$ is decreasing and $\beta(r, s) \rightarrow 0$ as $s \rightarrow \infty$.
} 
the eigenvalue with maximal absolute value) is real and nonnegative, and since $\exp (A t)$ is non-singular, it is in fact positive.

If both $A_{0}$ and $A_{1}$ are Metzler and $x(0) \in \mathbb{R}_{+}^{n}$ then (1) is called a positive linear switched system (PLSS). Mason and Shorten [17], and independently David Angeli, posed the following.

Conjecture 1: If (1) is a PLSS, then Assumption 1 provides a sufficient condition for GUAS.

Had this conjecture been true, it would have implied that determining GUAS for a PLSS is relatively simple. (See [18] for analysis of the computational complexity of determining whether any matrix in a convex set of matrices is Hurwitz.) Gurvits, Shorten, and Mason [19] proved that Conjecture 1 is in general false (see also [20]), but that it does hold when $n=2$ (even when the number of subsystems is arbitrary). Their proof in the planar case is based on showing that the PLSS admits a common quadratic Lyapunov function (CQLF). (For more on the analysis of switched systems using CQLFs, see [5], [4], [21], [22], [23].) Margaliot and Branicky [24] derived a reachability-with-nice-controls-type result for planar bilinear control systems, and showed that the proof of Conjecture 1 when $n=2$ follows as a special case. Fainshil, Margaliot, and Chigansky [25] showed that Conjecture 1 is false already for the case $n=3$. In general, it seems that as far as the GUAS problem is concerned, analyzing PLSSs is not simpler than analyzing linear switched systems.

There is a rich literature on sufficient conditions for GUAS, see, e.g., [5], [6], [8], [7], [12]. A more challenging problem is to determine a necessary and sufficient condition for GUAS. What makes this problem difficult is that the set of all possible switching laws is huge, so exhaustively checking the solution for each switching law is impossible.

A natural idea is to try and characterize a "most destabilizing" switching law $\sigma^{*}$ of the switched system, and then analyze the behavior of the corresponding trajectory $x^{*}$. If $x^{*}$ converges to the origin, then so does any trajectory of the switched system and this establishes GUAS. This idea was pioneered by E. S. Pyatntisky [26], [27], who studied the celebrated absolute stability problem (ASP). This variational approach was further developed by several scholars including N. E. Barabanov and L. B. Rapoport, and proved to be highly successful; see the survey papers [28], [29], [30], the related work in [31], [32], and the recent extensions to the stability analysis of discrete-time linear switched systems in [33], [34].

A first attempt to extend the variational approach to the stability analysis of PLSSs was taken in [35] using the classical Pontryagin maximum principle (PMP). Recently, Fainshil and Margaliot [2] developed an alternative approach that combines the Perron-Frobenius theory of nonnegative matrices with the 
standard needle variation used in the PMP.

The goal of this paper is to derive stronger, higher-order necessary conditions for optimality. We thus begin by reviewing the first-order MP in [2].

\section{A. Stability analysis of PLSSs: a Variational Approach}

The variational approach to the stability analysis of a linear switched system includes several steps. The first step is relaxing (1) to the bilinear control system (BCS)

$$
\begin{aligned}
\dot{x} & =(A+u B) x, \quad u \in \mathcal{U}, \\
x(0) & =x_{0},
\end{aligned}
$$

where $A:=\left(A_{0}+A_{1}\right) / 2, B:=\left(A_{1}-A_{0}\right) / 2$, and $\mathcal{U}$ is the set of measurable controls taking values in $[-1,1]$. Note that for $u(t) \equiv-1[u(t) \equiv 1]$, Eq. (4) yields $\dot{x}=A_{0} x\left[\dot{x}=A_{1} x\right]$, i.e., trajectories of the BCS corresponding to piecewise constant bang-bang controls are also trajectories of the original switched system.

The BCS (4) is said to be globally asymptotically stable (GAS) if $\lim _{t \rightarrow \infty} x(t)=0$ for all $x_{0} \in \mathbb{R}^{n}$ and all $u \in \mathcal{U}$. Since every trajectory of the switched system (1) is also a trajectory of (4), GAS of (4) implies GUAS of the linear switched system. It is not difficult to show that the converse implication also holds, so the BCS is GAS if and only if the linear switched system is GUAS. Thus, the GUAS problem for the switched linear system (1) is equivalent to the GAS problem for the BCS (4).

From here on we assume that the switched system is positive, i.e. $A+k B$ is Metzler for all $k \in[-1,1]$. For the BCS, this implies that if $x_{0} \in \mathbb{R}_{+}^{n}$, then $x(t) \in \mathbb{R}_{+}^{n}$ for all $u \in \mathcal{U}$ and all $t \geq 0$. Thus (4) becomes a positive bilinear control system (PBCS).

For $0 \leq a \leq b \leq T$, and $u \in \mathcal{U}$, let $C(b, a, u)$ denote the solution at time $t=b$ of the matrix differential equation

$$
\begin{aligned}
\frac{d}{d t} C(t, a, u) & =(A+B u(t)) C(t, a, u), \\
C(a, a, u) & =I .
\end{aligned}
$$

It is straightforward to verify that the solution of (4) satisfies $x(b)=C(b, a, u) x(a)$ for all $u \in \mathcal{U}$ and all $0 \leq a \leq b \leq T$. In other words, $C(b, a, u)$ is the transition matrix from time $a$ to time $b$ of (4) corresponding to the control $u$. To simplify the notation, we will sometimes omit the dependence on $u$ 
and just write $C(b, a)$.

When the initial time is $a=0$ we write (5) as

$$
\begin{aligned}
\dot{C}(t) & =(A+B u(t)) C(t), \\
C(0) & =I .
\end{aligned}
$$

For a $\mathrm{PBCS}, C(t, u)$ is a non-negative matrix for all $t \geq 0$ and all $u \in \mathcal{U}$. Since it is also non-singular, the spectral radius $\rho(C(t, u))$ is a real and positive eigenvalue of $C(t, u)$, called the Perron root. If this eigenvalue is simple then the corresponding eigenvector $v \in \mathbb{R}_{+}^{n}$, called the Perron eigenvector, is unique (up to multiplication by a scalar). The next step in the variational approach is to relate $\rho(C(t, u))$ to GAS of the PBCS.

Define the generalized spectral radius of the PBCS (4) by

$$
\rho(A, B):=\limsup _{t \rightarrow \infty} \rho_{t}(A, B)
$$

where

$$
\rho_{t}(A, B):=\max _{u \in \mathcal{U}}(\rho(C(t, u)))^{1 / t}
$$

Note that the maximum here is well-defined, as the reachable set of (6) corresponding to $\mathcal{U}$ is compact [36]. In fact, this is why we consider a bilinear control system with controls in $\mathcal{U}$ rather than the original linear switched system with piecewise constant switching laws.

The next result relates the GAS of the PBCS to $\rho(A, B)$.

Theorem 1: The PBCS (4) is GAS if and only if

$$
\rho(A, B)<1 \text {. }
$$

Thm. 1 already appeared in [2], but without a proof. For the sake of completeness we include its proof in the Appendix.

Remark 1: It follows from (7) and Thm. 11 that if $\rho(C(T, u)) \geq 1$ for some $T>0$ and $u \in \mathcal{U}$, then the PBCS is not GAS. Indeed, for any integer $k>0$, define $\bar{u}:[0, k T] \rightarrow[-1,1]$ via the periodic extension of $u$, and let $\bar{C}(t)$ denote the corresponding solution of (6) at time $t$. Then

$$
\rho(\bar{C}(k T))=(\rho(C(T)))^{k}
$$


so (7) yields

$$
\begin{aligned}
\rho_{k T}(A, B) & \geq(\rho(\bar{C}(k T)))^{1 /(k T)} \\
& =(\rho(C(T)))^{1 / T} \\
& \geq 1,
\end{aligned}
$$

and this implies that $\rho(A, B) \geq 1$.

Thm. 1 motivates the following optimal control problem.

Problem 1: Consider the PBCS (6). Fix an arbitrary final time $T>0$. Find a control $u^{*} \in \mathcal{U}$ that maximizes $\rho(C(T, u))$.

The main result in [2] is a first-order necessary condition for optimality. Let $A^{\prime}$ denote the transpose of the matrix $A$.

Theorem 2: [2] Consider the PBCS (6). Suppose that $u^{*} \in \mathcal{U}$ is an optimal control for Problem 1 . Let $C^{*}(t)$ denote the corresponding solution of (6) at time $t$, and let $\rho^{*}:=\rho\left(C^{*}(T)\right)$. Suppose that $\rho^{*}$ is a simple eigenvalue of $C^{*}(T)$. Let $v^{*} \in \mathbb{R}_{+}^{n}\left[w^{*} \in \mathbb{R}_{+}^{n}\right]$ be an eigenvector of $C^{*}(T)\left[\left(C^{*}(T)\right)^{\prime}\right]$ corresponding to $\rho^{*}$, normalized such that $\left(w^{*}\right)^{\prime} v^{*}=1$. Let $q:[0, T] \rightarrow \mathbb{R}_{+}^{n}$ be the solution of

$$
\begin{aligned}
\dot{q} & =-\left(A+B u^{*}\right)^{\prime} q, \\
q(T) & =w^{*},
\end{aligned}
$$

and let $p:[0, T] \rightarrow \mathbb{R}_{+}^{n}$ be the solution of

$$
\begin{aligned}
\dot{p} & =\left(A+B u^{*}\right) p, \\
p(0) & =v^{*} .
\end{aligned}
$$

Define the switching function $m:[0, T] \rightarrow \mathbb{R}$ by

$$
m(t):=q^{\prime}(t) B p(t) .
$$

Then for almost all $t \in[0, T]$,

$$
u^{*}(t)= \begin{cases}1, & m(t)>0 \\ -1, & m(t)<0\end{cases}
$$


This MP has some special properties.

Remark 2: First, note that (8) implies that

$$
q^{\prime}(0)=q^{\prime}(t) C^{*}(t), \quad \text { for all } t \in[0, T]
$$

In particular, substituting $t=T$ yields

$$
\begin{aligned}
q^{\prime}(0) & =q^{\prime}(T) C^{*}(T) \\
& =\left(w^{*}\right)^{\prime} C^{*}(T) \\
& =\rho^{*}\left(w^{*}\right)^{\prime},
\end{aligned}
$$

as $w^{*}$ is an eigenvector of $\left(C^{*}(T)\right)^{\prime}$ corresponding to the eigenvalue $\rho^{*}$. Since scaling $q$ by a positive constant has no effect on the sign of $m$, this means that the final condition $q(T)=w^{*}$ in (8) can be replaced by the initial condition $q(0)=w^{*}$. This leads to an MP in the form of a one-point boundary value problem (with the unknown $v^{*}, w^{*}$ as the initial conditions at time 0 ).

Remark 3: Note that

$$
\begin{aligned}
m(T) & =q^{\prime}(T) B p(T) \\
& =\left(w^{*}\right)^{\prime} B C^{*}(T) p(0) \\
& =\left(w^{*}\right)^{\prime} B \rho^{*} v^{*} \\
& =q^{\prime}(0) B p(0) \\
& =m(0)
\end{aligned}
$$

Thus, the switching function is "periodic" in the sense that $m(T)=m(0)$.

One difficulty in applying Theorem 2 is that both $v^{*}$ and $w^{*}$ are unknown. There are cases where this difficulty may be alleviated somewhat berceuse $w^{*}$ can be expressed in terms of $v^{*}$. The next example demonstrates this.

Example 1: Consider an optimal bang-bang control in the form

$$
u^{*}(t)= \begin{cases}1, & t \in\left(0, t_{1}\right) \\ -1, & t \in\left(t_{1}, T\right)\end{cases}
$$


where $0<t_{1}<T$. The corresponding transition matrix is

$$
C^{*}(T)=\exp \left((A-B) \tau_{2}\right) \exp \left((A+B) \tau_{1}\right)
$$

where $\tau_{1}:=t_{1}-0$ and $\tau_{2}:=T-t_{1}$. Thus, $v^{*}$ and $w^{*}$ satisfy

$$
\exp \left((A-B) \tau_{2}\right) \exp \left((A+B) \tau_{1}\right) v^{*}=\rho^{*} v^{*}
$$

and

$$
\exp \left((A+B)^{\prime} \tau_{1}\right) \exp \left((A-B)^{\prime} \tau_{2}\right) w^{*}=\rho^{*} w^{*}
$$

Suppose that $A$ and $B$ are symmetric matrices. Then (13) becomes

$$
\exp \left((A+B) \tau_{1}\right) \exp \left((A-B) \tau_{2}\right) w^{*}=\rho^{*} w^{*}
$$

and multiplying this on the left by $\exp \left((A-B) \tau_{2}\right)$ yields

$$
C^{*}(T) \exp \left((A-B) \tau_{2}\right) w^{*}=\rho^{*} \exp \left((A-B) \tau_{2}\right) w^{*}
$$

Since the Perron eigenvector of $C^{*}(T)$ is unique (up to multiplication by a constant) this means that

$$
\exp \left((A-B) \tau_{2}\right) w^{*}=r v^{*}
$$

for some $r>0$.

The MP in Theorem 2 is a necessary, but not sufficient, condition for optimality and it is possible of course that a control satisfying this MP is not an optimal control. The next example demonstrates this.

Example 2: Consider a PBCS satisfying the following properties:

- The matrix $A$ is symmetric. Its maximal eigenvalue $\mu$ is simple with corresponding eigenvector $z$, and

$$
z^{\prime} B z=0
$$

- The matrices $A-B$ and $A+B$ are Metzler;

- $\rho(A+B)>\rho(A)=\mu$.

(A specific example is $n=2, A=\left[\begin{array}{cc}2.2 & 1.6 \\ 1.6 & -0.2\end{array}\right]$ and $B=\left[\begin{array}{cc}-1.1 & 0.2 \\ 0.95 & 2.1\end{array}\right]$. Indeed, here $\mu=3, z=\left[\begin{array}{ll}2 & 1\end{array}\right]^{\prime}$ 
and it is straightforward to verify that all the properties above hold.)

Consider the possibility that the singular control $u(t) \equiv 0$ is optimal. Then

$$
\rho(u):=\rho(\exp (A T))=\exp (\mu T)
$$

Since $A$ is symmetric, the corresponding right and left eigenvector is $z$, so in the MP $p(0)=q(T)=z$. Thus, (9) and (8) yield

$$
\begin{aligned}
p(t) & =\exp (A t) z \\
& =\exp (\mu t) z,
\end{aligned}
$$

and

$$
\begin{aligned}
q(t) & =\exp \left(A^{\prime}(T-t)\right) z \\
& =\exp (\mu(T-t)) z
\end{aligned}
$$

Substituting this in (10) yields

$$
m(t)=\exp (\mu T) z^{\prime} B z \equiv 0
$$

Thus, $u(t) \equiv 0$ (vacuously) satisfies Thm. 2. However, since $\rho(A+B)>\rho(A)$ the control $\tilde{u}(t) \equiv 1$ yields

$$
\rho(\tilde{u}):=\exp ((A+B) T)>\rho(u)
$$

so clearly $u(t) \equiv 0$ is not an optimal control.

The reason that $u(t) \equiv 0$ in Example 2 cannot be ruled out is that Thm. 2 is a first-order MP. More specifically, its derivation is based the following idea. Suppose that $u$ is a candidate for an optimal control. Introduce a new control $\tilde{u}:[0, T] \rightarrow[-1,1]$ by adding a needle variation to $u$, i.e.

$$
\tilde{u}(t):= \begin{cases}a, & t \in[\tau, \tau+\epsilon), \\ u(t), & \text { otherwise, }\end{cases}
$$

where $a \in[-1,1], \tau \in[0, T)$ is a Lebesgue point of $u$, and $\epsilon>0$ is sufficiently small, and analyze the 
difference $C(T, \tilde{u})-C\left(T, u^{*}\right)$ to first-order in $\epsilon$. For $u(t) \equiv 0$,

$$
C(T, \tilde{u})=\exp (A(T-\tau-\epsilon)) \exp ((A+a B) \epsilon) \exp (A \tau)
$$

so

$$
\left.\frac{d}{d \epsilon} C(T, \tilde{u})\right|_{\epsilon=0}=a \exp (A(T-\tau)) B \exp (A \tau)
$$

Combining this with known results on the derivative of a simple eigenvalue of a matrix (see, e.g. [37, Chapter 6]) yields

$$
\rho(C(T, \tilde{u}))=\rho(C(T, u))+\epsilon a w^{\prime} \exp (A(T-\tau)) B \exp (A \tau) v+o(\epsilon)
$$

If $a w^{\prime} \exp (A(T-\tau)) B \exp (A \tau) v>0$ then $\rho(C(T, \tilde{u}))>\rho(C(T, u))$ for all sufficiently small $\epsilon>0$ and thus $u$ is not optimal. However, in Example 2 the term multiplying $\epsilon$ in (15) is zero for all $a, \tau$, and $T$, so a first-order analysis cannot rule out the possibility that $u$ is optimal.

Summarizing, Example 2 suggests that there is a need for a higher-order MP, i.e., an MP that takes into account higher-order terms in the Taylor expansion of $\rho(C(T, \tilde{u}))-\rho(C(T, u))$ with respect to $\epsilon$, and can thus be used to rule out the optimality of a larger set of controls.

In the next section, we apply the generalized Legendre-Clebsch condition to derive a high-order necessary condition for a singular control to be optimal. We also combine known results on the secondorder derivative of the Perron root [38] and the Agrachev-Gamkrelidze second-order variation for bangbang controls (see, e.g., [39]) to derive a second-order MP for bang-bang controls. The proofs of these results are given in Section

\section{MAin RESUlts}

Our first result is a high-order necessary condition for singular optimal controls for Problem 1 , Without loss of generality (see [40]), we assume that the singular control is $u^{*}(t) \equiv 0$. Let $[P, Q]:=Q P-P Q$ denote the Lie-bracket of the matrices $P, Q \in \mathbb{R}^{n \times n}$.

\section{A. High-order MP for singular controls}

Theorem 3: Consider the PBCS (4). Suppose that the conditions of Thm. 2 hold, and that $u^{*}(t) \equiv 0$ is an optimal control. Then

$$
\left(w^{*}\right)^{\prime}[B,[B, A]] v^{*} \leq 0
$$


Example 3: Consider the specific PBCS with $n=2$ given in Example 2. In this case,

$$
[B,[B, A]]=\left[\begin{array}{cc}
6.8 & 18.4 \\
21.4 & -6.8
\end{array}\right]
$$

and $v^{*}=w^{*}=\left[\begin{array}{ll}2 & 1\end{array}\right]^{\prime} / \sqrt{5}$, so

$$
\left(w^{*}\right)^{\prime}[B,[B, A]] v^{*}=20
$$

It follows from (16) that $u^{*}(t) \equiv 0$ is not an optimal control. Note that we were not able to derive this conclusion using the first-order MP in Thm. 2.

\section{B. Second-order MP for bang-bang controls}

In this section, we derive an Agrachev-Gamkrelidze-type second-order MP for optimal bang-bang controls for Problem 1. Note that for an optimal bang-bang $u^{*}$ we have

$$
C^{*}(T)=\exp \left((A+B) \tau_{k}\right) \ldots \exp \left((A+B) \tau_{2}\right) \exp \left((A-B) \tau_{1}\right)
$$

with $\tau_{i} \geq 0$ and $\sum_{i=1}^{k} \tau_{i}=T$. Any cyclic shift of $C^{*}(T)$, e.g.,

$$
\exp \left((A-B) \tau_{1}\right) \exp \left((A+B) \tau_{k}\right) \ldots \exp \left((A+B) \tau_{2}\right)
$$

also corresponds to an optimal control (as a product of matrices and its cyclic shift have the same spectral radius). This means that we can always assume that $t_{0}:=0$ is a switching point of $u^{*}$, and then (12) implies that $T$ is also a switching point of $u^{*}$.

Let $\mathcal{P}^{k}$ denote the set of all vectors $\alpha=\left[\begin{array}{llll}\alpha_{0} & \alpha_{1} & \ldots & \alpha_{k}\end{array}\right]^{\prime} \in \mathbb{R}^{k+1}$ satisfying

$$
\alpha_{1}+\cdots+\alpha_{k}=0
$$

We can now state the main result in this section.

Theorem 4: Suppose that $u^{*}$ is an optimal control for Problem 1, that the conditions of Thm. 2 hold, and that the switching function (10) admits a finite number of zeros at $t_{0}<t_{1}<\cdots<t_{k}$, with $t_{0}=0, t_{k}=T$, so that $u^{*}(t)=r$ for $t \in\left(0, t_{1}\right), u^{*}(t)=-r$ for $t \in\left(t_{1}, t_{2}\right), u^{*}(t)=r$ for $t \in\left(t_{2}, t_{3}\right)$, and so on, with $r \in\{-1,1\}$. Denote $P:=A+r B, Q:=A-r B$, and $\tau_{i}:=t_{i}-t_{i-1}$. Define matrices $H_{i} \in \mathbb{R}^{n \times n}$, 
$i=1, \ldots, k$, by

$$
\begin{aligned}
& H_{1}:=P, \\
& H_{2}:=Q \\
& H_{3}:=\exp \left(-\tau_{2} Q\right) P \exp \left(\tau_{2} Q\right), \\
& H_{4}:=\exp \left(-\tau_{2} Q\right) \exp \left(-\tau_{3} P\right) Q \exp \left(\tau_{3} P\right) \exp \left(\tau_{2} Q\right), \\
& H_{5}:=\exp \left(-\tau_{2} Q\right) \exp \left(-\tau_{3} P\right) \exp \left(-\tau_{4} Q\right) P \exp \left(\tau_{4} Q\right) \exp \left(\tau_{3} P\right) \exp \left(\tau_{2} Q\right),
\end{aligned}
$$

Then

$$
q^{\prime}\left(t_{1}\right) \sum_{i=1}^{k} \alpha_{i} H_{i} p\left(t_{1}\right)=0, \quad \text { for all } \alpha \in \mathcal{P}^{k}
$$

Furthermore,

$$
r_{k}(\alpha):=q^{\prime}\left(t_{1}\right) \sum_{1 \leq i<j \leq k} \alpha_{i} \alpha_{j}\left[H_{i}, H_{j}\right] p\left(t_{1}\right)
$$

satisfies

$$
r_{k}(\alpha) \leq 0, \quad \text { for all } \alpha \in \mathcal{Q}^{k}
$$

where

$$
\mathcal{Q}^{k}:=\left\{\alpha \in \mathcal{P}^{k}: \sum_{i=1}^{k} \alpha_{i} H_{i} p\left(t_{1}\right)=0\right\} .
$$

We refer to the control $u^{*}$ defined above as a control with $k$ bang arcs. As will be shown in the proof, condition (19) is a first-order condition (that can also be derived using the first-order MP). Condition (20) however is a second-order condition, and it is meaningful for values $\alpha$ that make a certain first-order variation vanish, i.e. that belong to $\mathcal{Q}^{k}$.

Note that the conditions in Thm. 4 are given in terms of $p\left(t_{1}\right)$ and $q\left(t_{1}\right)$. It is possible of course to state them in terms of $p\left(t_{0}\right)=v^{*}$ and $q\left(t_{0}\right)=\rho^{*} w^{*}$, but this leads to slightly more cumbersome expressions.

The next example demonstrates the calculations for a control with two bang arcs.

Example 4: Consider an optimal control in the form

$$
u^{*}(t)= \begin{cases}1, & t \in\left(0, t_{1}\right) \\ -1, & t \in\left(t_{1}, T\right)\end{cases}
$$


where $0<t_{1}<T$. In this case, (19) becomes

$$
q^{\prime}\left(t_{1}\right)\left(\alpha_{1}(A+B)+\alpha_{2}(A-B)\right) p\left(t_{1}\right)=0, \quad \text { for all } \alpha \in \mathcal{P}^{2}
$$

and the definition of $\mathcal{P}^{2}$ yields

$$
\alpha_{1} q^{\prime}\left(t_{1}\right)((A+B)-(A-B)) p\left(t_{1}\right)=0, \quad \text { for all } \alpha_{1} \in \mathbb{R}
$$

Of course, this is just the conclusion that we can get from the first-order MP, as at the switching point $t_{1}$ we must have

$$
0=m\left(t_{1}\right)=q^{\prime}\left(t_{1}\right) B p\left(t_{1}\right)
$$

The second-order term is

$$
\begin{aligned}
r_{2}(\alpha) & =\alpha_{1} \alpha_{2} q^{\prime}\left(t_{1}\right)\left[H_{1}, H_{2}\right] p\left(t_{1}\right) \\
& =-\alpha_{1}^{2} q^{\prime}\left(t_{1}\right)[A+B, A-B] p\left(t_{1}\right) \\
& =2 \alpha_{1}^{2} q^{\prime}\left(t_{1}\right)[A, B] p\left(t_{1}\right),
\end{aligned}
$$

so (20) becomes

$$
r_{2}(\alpha) \leq 0, \quad \text { for all } \alpha \in \mathcal{Q}^{2}
$$

where

$$
\mathcal{Q}^{2}=\left\{\alpha_{1} \in \mathbb{R}: \alpha_{1} B p\left(t_{1}\right)=0\right\}
$$

Again, this provides information that can also be derived from the first-order MP, as the fact that $m\left(t_{1}^{-}\right)>0$ and $m\left(t_{1}^{+}\right)<0$ implies that

$$
\dot{m}\left(t_{1}\right) \leq 0
$$

and differentiating (10) yields

$$
\dot{m}\left(t_{1}\right)=q^{\prime}\left(t_{1}\right)[A, B] p\left(t_{1}\right) \text {. }
$$

Thus, $q^{\prime}\left(t_{1}\right)[A, B] p\left(t_{1}\right) \leq 0$, so (22) actually holds for all $\alpha \in \mathcal{P}^{2}$.

However, for a control with more than two bang arcs the second-order condition does provide new information. The next simple example demonstrates this. 
Example 5: Consider the PBCS (6) with

$$
A=\left[\begin{array}{cc}
-5 / 2 & 3 / 2 \\
3 & -5 / 2
\end{array}\right], \quad B=\left[\begin{array}{cc}
3 / 2 & -1 / 2 \\
1 & -3 / 2
\end{array}\right] \text {. }
$$

Note that $A+k B$ is Metzler for all $k \in[-1,1]$. Consider the control

$$
u(t)= \begin{cases}1, & t \in\left(0, t_{1}\right) \cup\left(t_{2}, t_{3}\right), \\ -1, & t \in\left(t_{1}, t_{2}\right) \cup\left(t_{3}, T\right),\end{cases}
$$

with $t_{1}=1, t_{2}=2, t_{3}=3$, and $T=4$. The corresponding transition matrix is

$$
C(T)=\exp (A-B) \exp (A+B) \exp (A-B) \exp (A+B)
$$

Let $s:=(9+32 \exp (5)+9 \exp (10))^{1 / 2}$. The spectral radius of $C(T)$ is

$$
\rho=\left(\frac{9+7 \exp (5)+9 \exp (10)+3 s(\exp (5)-1)}{25 \exp (10)}\right)^{2},
$$

and it is a simple eigenvalue. The Perron right and left eigenvectors of $C(T)$ are

$$
v=[\exp (5)-1+s \quad 2+8 \exp (5)]^{\prime}
$$

and

$$
w=[\exp (5)-1+s \quad 4+\exp (5)]^{\prime}
$$

Calculating the switching function $m$ defined in (10) yields the behavior depicted in Fig. 11. Note that $m(t)>$ 0 for $t \in\left(0, t_{1}\right) \cup\left(t_{2}, t_{3}\right)$, and $m(t)<0$ for $t \in\left(t_{1}, t_{2}\right) \cup\left(t_{3}, T\right)$, so the control $u$ satisfies the first-order MP.

We now show that the second-order MP implies that $u$ is not an optimal control. Eq. (18) yields

$$
\begin{aligned}
& H_{1}=A+B \\
& H_{2}=A-B \\
& H_{3}=\exp (-(A-B))(A+B) \exp (A-B), \\
& H_{4}=\exp (-(A-B)) \exp (-(A+B))(A-B) \exp (A+B) \exp (A-B) .
\end{aligned}
$$


Note that

$$
\begin{aligned}
H_{3} & =\exp (-(A-B))(A-B+2 B) \exp (A-B) \\
& =A-B+2 \exp (-(A-B)) B \exp (A-B) .
\end{aligned}
$$

Our goal is to find $\bar{\alpha} \in \mathcal{Q}^{4}$ such that $r_{4}(\bar{\alpha})>0$. Indeed, this will imply that $u$ is not optimal. It turns out that we can find such an $\bar{\alpha}$ satisfying $\bar{\alpha}_{1}=1$ and $\bar{\alpha}_{4}=0$. Since $\sum_{i=1}^{4} \bar{\alpha}_{i}$ must be zero, $\bar{\alpha}_{3}=-1-\bar{\alpha}_{2}$. Then

$$
\begin{aligned}
\sum_{i=1}^{4} \bar{\alpha}_{i} H_{i} & =A+B+\bar{\alpha}_{2}(A-B)-\left(1+\bar{\alpha}_{2}\right)(A-B+2 \exp (-(A-B)) B \exp (A-B)) \\
& =2 B-2\left(1+\bar{\alpha}_{2}\right) \exp (-(A-B)) B \exp (A-B),
\end{aligned}
$$

so

$$
\begin{aligned}
\sum_{i=1}^{4} \bar{\alpha}_{i} H_{i} p\left(t_{1}\right) & =2\left(B-\left(1+\bar{\alpha}_{2}\right) \exp (-(A-B)) B \exp (A-B)\right) \exp (A+B) v \\
& =2 B \exp (A+B) v-2 \sqrt{\rho}\left(1+\bar{\alpha}_{2}\right) \exp (-(A-B)) B v
\end{aligned}
$$

A tedious but straightforward calculation shows that

$$
B \exp (A+B) v=\exp (-5) \exp (-(A-B)) B v
$$

so $\sum_{i=1}^{4} \bar{\alpha}_{i} H_{i} p\left(t_{1}\right)=0$ for

$$
\bar{\alpha}_{2}=(\sqrt{\rho} \exp (5))^{-1}-1
$$

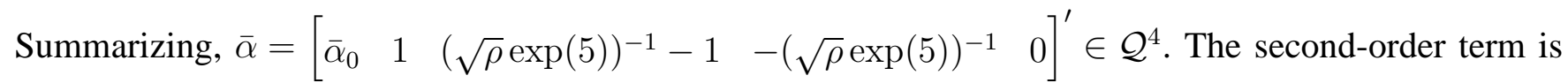

$$
\begin{aligned}
r_{4}(\bar{\alpha}) & =q^{\prime}\left(t_{1}\right) \sum_{1 \leq i<j \leq 4} \bar{\alpha}_{i} \bar{\alpha}_{j}\left[H_{i}, H_{j}\right] p\left(t_{1}\right) \\
& =q^{\prime}\left(t_{1}\right)\left(\bar{\alpha}_{2}\left[H_{1}, H_{2}\right]+\bar{\alpha}_{3}\left[H_{1}, H_{3}\right]+\bar{\alpha}_{2} \bar{\alpha}_{3}\left[H_{2}, H_{3}\right]\right) p\left(t_{1}\right),
\end{aligned}
$$

and a calculation yields

$$
r_{4}(\bar{\alpha})=\frac{1050 \exp (5)(\exp (5)-1)(12(s-3)+\exp (5)(-67+s+\exp (5)(67+36 \exp (5)+12 s)))}{(4+\exp (5))(1+4 \exp (5))(9-3 s+\exp (5)(7+9 \exp (5)+3 s))^{2}}
$$

Clearly, $r_{4}(\bar{\alpha})>0$, so the second-order MP implies that $u$ in $(23)$ is not an optimal control. The reason 


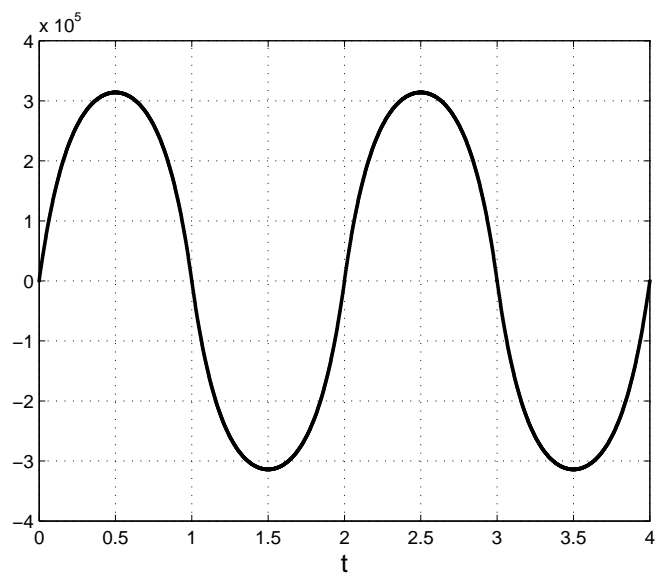

Fig. 1. Switching function $m(t)$ in Example 5

that $u$ here satisfies the conditions of the first-order MP is that it actually minimizes the spectral radius at time $T=4$. Thus, the second-order MP plays here a similar role to the second-derivative of a function: it allows to distinguish between a maximum point and a minimum point.

\section{PROOFS}

\section{A. Proof of Thm. 3}

Assume that $u^{*}(t) \equiv 0$ is an optimal control. The corresponding solution of (6) is $C^{*}(T)=\exp (A T)$. For $\epsilon>0$, consider the control

$$
\tilde{u}(t):= \begin{cases}0, & t \in\left[0, T-4 \epsilon^{1 / 3}\right), \\ -1, & t \in\left[T-4 \epsilon^{1 / 3}, T-3 \epsilon^{1 / 3}\right), \\ 1, & t \in\left[T-3 \epsilon^{1 / 3}, T-\epsilon^{1 / 3}\right), \\ -1, & t \in\left[T-\epsilon^{1 / 3}, T\right) .\end{cases}
$$

Then

$$
\begin{aligned}
C(T, \tilde{u}) & =\exp \left((A-B) \epsilon^{1 / 3}\right) \exp \left((A+B) 2 \epsilon^{1 / 3}\right) \exp \left((A-B) \epsilon^{1 / 3}\right) \exp \left(A\left(T-4 \epsilon^{1 / 3}\right)\right) \\
& =\exp \left((A-B) \epsilon^{1 / 3}\right) \exp \left((A+B) 2 \epsilon^{1 / 3}\right) \exp \left((A-B) \epsilon^{1 / 3}\right) \exp \left(-4 \epsilon^{1 / 3} A\right) C^{*}(T),
\end{aligned}
$$

and it follows from the computation in [40, p. 719] (see also [41]) that

$$
C(T, \tilde{u})=\exp \left(\frac{2 \epsilon}{3}[B,[B, A]]\right) C^{*}(T)+o(\epsilon) .
$$


Note that this implies that any result derived using $\tilde{u}$ will be a high-order MP, as the width of the needle variations in (25) is of order $\epsilon^{1 / 3}$ yet the perturbation in $C(T, \tilde{u})$ with respect to $C^{*}(T)$ is of order $\epsilon$. By (26),

$$
\left.\frac{d}{d \epsilon} C(T, \tilde{u})\right|_{\epsilon=0}=(2 / 3)[B,[B, A]] C^{*}(T),
$$

so

$$
\begin{aligned}
\rho(C(T, \tilde{u})) & -\rho\left(C^{*}(T)\right) \\
& =(2 \epsilon / 3)\left(w^{*}\right)^{\prime}[B,[B, A]] C^{*}(T) v^{*}+o(\epsilon) \\
& =(2 \epsilon / 3)\left(w^{*}\right)^{\prime}[B,[B, A]] \rho\left(C^{*}(T)\right) v^{*}+o(\epsilon) .
\end{aligned}
$$

If $\left(w^{*}\right)^{\prime}[B,[B, A]] v^{*}>0$ then $\rho(C(T, \tilde{u}))>\rho\left(C^{*}(T)\right)$ for all sufficiently small $\epsilon>0$, and this contradicts the optimality of $u^{*}$. This proves (16).

\section{B. Proof of Theorem 4}

The proof is based on introducing a new control defined by a perturbation of the switching times $t_{0}, \ldots, t_{k}$ to $\tilde{t}_{0}:=t_{0}+s \alpha_{0}, \tilde{t}_{1}:=t_{1}+s\left(\alpha_{0}+\alpha_{1}\right), \ldots, \tilde{t}_{k}:=t_{k}+s\left(\alpha_{0}+\alpha_{1}+\cdots+\alpha_{k}\right)$. Here, $s \in \mathbb{R}$ and $\alpha \in \mathcal{P}^{k}$. Define $\tilde{u}(t ; s, \alpha)$ by $\tilde{u}(t)=r$ for $t \in\left(\tilde{t}_{0}, \tilde{t}_{1}\right), \tilde{u}(t)=-r$ for $t \in\left(\tilde{t}_{1}, \tilde{t}_{2}\right)$, and so on. Note that (17) implies that the time length of the perturbed control is

$$
\tilde{t}_{k}-\tilde{t}_{0}=t_{k}+s\left(\alpha_{1}+\ldots \alpha_{k}\right)-t_{0}=t_{k}-t_{0}=T
$$

Denote the corresponding transition matrix by $\tilde{C}(t)=\tilde{C}(t ; s, \alpha)$. Note also that $\tilde{u}(\cdot ; 0, \alpha)=u^{*}(\cdot)$ for any $\alpha$, so $\tilde{C}(\cdot ; 0, \alpha)=C^{*}(\cdot)$. Our goal is to derive an expression for the difference $e(s, \alpha):=\rho(\tilde{C}(T ; s, \alpha))-$ $\rho\left(C^{*}(T)\right)$ in the form

$$
e(s, \alpha)=s z_{1}(\alpha)+\frac{s^{2}}{2} z_{2}(\alpha)+o\left(s^{2}\right)
$$

where $o\left(s^{2}\right)$ denotes a function $f$ that satisfies $\lim _{s \rightarrow 0} \frac{f(s)}{s^{2}}=0$.

Suppose for a moment that $z_{1}(\alpha)>0\left[z_{1}(\alpha)<0\right]$ for some $\alpha \in \mathcal{P}^{k}$. Then for any sufficiently small $s>0[s<0]$, (27) implies that $\rho(C(T ; s, \alpha))>\rho\left(C^{*}(T)\right)$. This contradicts the optimality of $u^{*}$, so

$$
z_{1}(\alpha)=0, \quad \text { for all } \alpha \in \mathcal{P}^{k}
$$


Thus, $e(s, \alpha)=\frac{s^{2}}{2} z_{2}(\alpha)+o\left(s^{2}\right)$, and a similar argument implies that the second-order term must satisfy $z_{2}(\alpha) \leq 0$. As we will see below, these conditions lead to the first- and second-order optimality conditions (19) and (20).

The calculation of the terms $z_{1}$ and $z_{2}$ in (27) requires two steps. The first is to derive an expression for the first- and second-order derivative of $\tilde{C}(T ; s, \alpha)$ with respect to $s$. This is based on the AgrachevGamkrelidze second-order variation for bang-bang controls [39] (see also [42], [43]). The second step is to derive an expression for the first- and second-order derivatives of the spectral radius of a matrix with respect to perturbations of the matrix entries. This follows the approach in [38].

\section{First- and second-order derivatives of the transition matrix}

From here on we consider the case where $k$ is even. (The derivations in the case where $k$ is odd are similar.) Thus,

$$
\tilde{C}(T ; s, \alpha)=\exp \left(\tilde{\tau}_{k} Q\right) \exp \left(\tilde{\tau}_{k-1} P\right) \ldots \exp \left(\tilde{\tau}_{2} Q\right) \exp \left(\tilde{\tau}_{1} P\right)
$$

where $\tilde{\tau}_{i}:=\tilde{t}_{i}-\tilde{t}_{i-1}=\tau_{i}+s \alpha_{i}$. Let $\dot{\tilde{C}}:=\frac{d}{d s} \tilde{C}$.

Proposition 1: The first- and second-order derivatives of $\tilde{C}(T ; s, \alpha)$ with respect to $s$ satisfy

$$
\begin{aligned}
\tilde{H} \dot{\tilde{C}} & =\sum_{i=1}^{k} \alpha_{i} \tilde{G}_{i} \\
\tilde{H} \ddot{\tilde{C}} & =-\dot{\tilde{H}} \dot{\tilde{C}}+\sum_{1 \leq i<j \leq k} \alpha_{i} \alpha_{j}\left[\tilde{G}_{i}, \tilde{G}_{j}\right]
\end{aligned}
$$

where

$$
\begin{aligned}
& \tilde{G}_{1}:=\exp \left(-\tilde{\tau}_{1} P\right) P \exp \left(\tilde{\tau}_{1} P\right)=P, \\
& \tilde{G}_{2}:=\exp \left(-\tilde{\tau}_{1} P\right) Q \exp \left(\tilde{\tau}_{1} P\right), \\
& \tilde{G}_{3}:=\exp \left(-\tilde{\tau}_{1} P\right) \exp \left(-\tilde{\tau}_{2} Q\right) P \exp \left(\tilde{\tau}_{2} Q\right) \exp \left(\tilde{\tau}_{1} P\right),
\end{aligned}
$$

and $\tilde{H}=\tilde{H}(s, \alpha):=\exp \left(-\tilde{\tau}_{1} P\right) \exp \left(-\tilde{\tau}_{2} Q\right) \ldots \exp \left(-\tilde{\tau}_{k} Q\right)$.

Proof. Rewrite (29) as

$$
I=\exp \left(-\tilde{\tau}_{1} P\right) \exp \left(-\tilde{\tau}_{2} Q\right) \ldots \exp \left(-\tilde{\tau}_{k-1} P\right) \exp \left(-\tilde{\tau}_{k} Q\right) \tilde{C}
$$


Differentiating both sides with respect to $s$ and rearranging terms yields

$$
\begin{aligned}
\tilde{H} \dot{\tilde{C}} & =\alpha_{1} P \tilde{C}_{0} \\
& +\alpha_{2} \exp \left(-\tilde{\tau}_{1} P\right) Q \tilde{C}_{1} \\
& +\alpha_{3} \exp \left(-\tilde{\tau}_{1} P\right) \exp \left(-\tilde{\tau}_{2} Q\right) P \tilde{C}_{2} \\
& \vdots \\
& +\alpha_{k} \exp \left(-\tilde{\tau}_{1} P\right) \exp \left(-\tilde{\tau}_{2} Q\right) \ldots \exp \left(-\tilde{\tau}_{k-1} P\right) Q \tilde{C}_{k-1},
\end{aligned}
$$

where $\tilde{C}_{0}:=I, \tilde{C}_{1}:=\tilde{C}\left(\tilde{\tau}_{1}\right), \tilde{C}_{2}=\tilde{C}\left(\tilde{\tau}_{1}+\tilde{\tau}_{2}\right)$, and so on. This yields the first equation in (30). Differentiating (32) with respect to $s$ yields

$$
\dot{\tilde{H}} \dot{\tilde{C}}+\tilde{H} \ddot{\tilde{C}}=\sum_{i=1}^{k-1} \sum_{j=i+1}^{k} \alpha_{i} \alpha_{j}\left[\tilde{G}_{i}, \tilde{G}_{j}\right],
$$

and this completes the proof of Prop. 1

The next step is to determine the first- and second-order derivatives of $\tilde{\rho}(s, \alpha)=\rho(\tilde{C}(T ; s, \alpha))$ with respect to $s$.

\section{First- and second-order derivatives of the spectral radius}

Let $\tilde{v}=\tilde{v}(s, \alpha), \tilde{w}=\tilde{w}(s, \alpha)$ denote a nonnegative right and a left eigenvector of $\tilde{C}(T)$ corresponding to the eigenvalue $\tilde{\rho}$, and normalized so that $\tilde{w}^{\prime} \tilde{v}=1$. Note that since $\rho^{*}$ is simple, the spectral radius of $\tilde{C}(T)$ will also be simple for all $|s|$ sufficiently small. For a matrix $D$, let $D^{\#}$ denote the Drazin inverse of $D$.

Proposition 2: The first- and second-order derivatives of $\tilde{\rho}$ with respect to $s$ satisfy

$$
\begin{aligned}
\left.\dot{\tilde{\rho}}\right|_{s=0} & =\left(w^{*}\right)^{\prime}\left(\left.\dot{\tilde{C}}\right|_{s=0}\right) v^{*} \\
\left.\ddot{\tilde{\rho}}\right|_{s=0} & =\left(w^{*}\right)^{\prime}\left(\left.\ddot{\tilde{C}}\right|_{s=0}\right) v^{*} \\
& +2\left(w^{*}\right)^{\prime}\left(\left.\dot{\tilde{C}}\right|_{s=0}\right)\left(\rho^{*} I-C^{*}\right)^{\#}\left(\left.\dot{\tilde{C}}\right|_{s=0}\right) v^{*}
\end{aligned}
$$

Proof. Differentiating the equation $\tilde{C} \tilde{v}=\tilde{\rho} \tilde{v}$ with respect to $s$ yields

$$
\dot{\tilde{C}} \tilde{v}+\tilde{C} \dot{\tilde{v}}=\dot{\tilde{\rho}} \tilde{v}+\tilde{\rho} \tilde{\tilde{v}}
$$


Multiplying on the left by $\tilde{w}^{\prime}$ and using the fact that $\tilde{w}^{\prime} \tilde{C}=\tilde{\rho} \tilde{w}^{\prime}$ yields

$$
\tilde{w}^{\prime} \dot{\tilde{C}} \tilde{v}+\tilde{\rho} \tilde{w}^{\prime} \dot{\tilde{v}}=\dot{\tilde{\rho}}+\tilde{\rho} \tilde{w}^{\prime} \dot{\tilde{v}}
$$

so

$$
\dot{\tilde{\rho}}=\tilde{w}^{\prime} \dot{\tilde{C}} \tilde{v}
$$

This proves (33). To calculate the second-order derivative, differentiate (34) with respect to $s$. This yields

$$
\ddot{\tilde{C}} \tilde{v}+2 \dot{\tilde{C}} \dot{\tilde{v}}+\tilde{C} \ddot{\tilde{v}}=\ddot{\tilde{\rho}} \tilde{v}+2 \dot{\tilde{\rho}} \dot{\tilde{v}}+\tilde{\rho} \ddot{\tilde{v}}
$$

Multiplying on the left by $\tilde{w}^{\prime}$ yields

$$
\tilde{w}^{\prime} \ddot{\tilde{C}} \tilde{v}+2 \tilde{w}^{\prime} \dot{\tilde{C}} \dot{\tilde{v}}+\tilde{\rho} \tilde{w}^{\prime} \ddot{\tilde{v}}=\ddot{\tilde{\rho}}+2 \dot{\tilde{\rho}} \tilde{w}^{\prime} \dot{\tilde{v}}+\tilde{\rho} \tilde{w}^{\prime} \ddot{\tilde{v}}
$$

so

$$
\begin{aligned}
\ddot{\tilde{\rho}} & =\tilde{w}^{\prime} \ddot{\tilde{C}} \tilde{v}+2 \tilde{w}^{\prime} \dot{\tilde{C}} \dot{\tilde{v}}-2 \dot{\tilde{\rho}} \tilde{w}^{\prime} \dot{\tilde{v}} \\
& =\tilde{w}^{\prime} \ddot{\tilde{C}} \tilde{v}+2 \tilde{w}^{\prime} \dot{\tilde{C}} \dot{\tilde{v}}-2\left(\tilde{w}^{\prime} \dot{\tilde{C}} \tilde{v}\right) \tilde{w}^{\prime} \dot{\tilde{v}} \\
& =\tilde{w}^{\prime} \ddot{\tilde{C}} \tilde{v}+2 \tilde{w}^{\prime} \dot{\tilde{C}}\left(I-\tilde{v} \tilde{w}^{\prime}\right) \dot{\tilde{v}}
\end{aligned}
$$

where the second equation follows from (35). To simplify this expression, let $\tilde{D}:=\tilde{\rho} I-\tilde{C}$. It follows from (34) that

$$
\tilde{D} \dot{\tilde{v}}=\dot{\tilde{C}} \tilde{v}-\dot{\tilde{\rho}} \tilde{v}
$$

Since $\rho^{*}$ is a simple eigenvalue of $C^{*}, \tilde{\rho}$ is a simple eigenvalue of $\tilde{C}$ for all $|s|$ sufficiently small, so zero is a simple eigenvalue of $\tilde{D}$. Hence, there exists a unique generalized inverse $\tilde{D}^{\#}$ of $\tilde{D}$ satisfying

$$
\tilde{D} \tilde{D}^{\#}=\tilde{D}^{\#} \tilde{D}, \quad \tilde{D} \tilde{D}^{\#} \tilde{D}=\tilde{D}, \quad \tilde{D}^{\#} \tilde{D} \tilde{D}^{\#}=\tilde{D}^{\#}
$$

Multiplying (37) on the left by $\tilde{D}^{\#}$ and using the fact that $\tilde{D}^{\#} \tilde{v}=0$ and (38) yields

$$
\tilde{D} \tilde{D}^{\#} \dot{\tilde{v}}=\tilde{D}^{\#} \dot{\tilde{C}} \tilde{v}
$$


It is not difficult to show that $I-\tilde{D} \tilde{D}^{\#}=\tilde{v} \tilde{w}^{\prime}$, so

$$
\left(I-\tilde{v} \tilde{w}^{\prime}\right) \dot{\tilde{v}}=\tilde{D} \# \dot{\tilde{C}} \tilde{v}
$$

Multiplying this on the left by $\tilde{w}^{\prime} \dot{\tilde{C}}$ yields

$$
\tilde{w}^{\prime} \dot{\tilde{C}}\left(I-\tilde{v} \tilde{w}^{\prime}\right) \dot{\tilde{v}}=\tilde{w}^{\prime} \dot{\tilde{C}} \tilde{D}^{\#} \dot{\tilde{C}} \tilde{v}
$$

and substituting this in (36) yields $\ddot{\tilde{\rho}}=\tilde{w}^{\prime} \ddot{\tilde{C}} \tilde{v}+2 \tilde{w}^{\prime} \dot{\tilde{C}} \tilde{D} \# \dot{\tilde{C}} \tilde{v}$. Setting $s=0$ completes the proof of Prop. 2.

We can now prove Thm. 4. Combining (27), (30), and (33) yields

$$
z_{1}(\alpha)=\sum_{i=1}^{k} \alpha_{i}\left(w^{*}\right)^{\prime}(H(0, \alpha))^{-1} G_{i} v^{*}
$$

where $G_{i}:=\left.\tilde{G}_{i}\right|_{s=0}$. It follows from (31) and (19) that $G_{i}=\exp \left(-\tau_{1} P\right) H_{i} \exp \left(\tau_{1} P\right)$. The definition of $q$ in (8) implies that

$$
\left(w^{*}\right)^{\prime}=q^{\prime}(T)=q^{\prime}(0) H(0, \alpha)
$$

So

$$
\begin{aligned}
z_{1}(\alpha) & =\sum_{i=1}^{k} \alpha_{i} q^{\prime}(0) G_{i} p(0) \\
& =\sum_{i=1}^{k} \alpha_{i} q^{\prime}\left(t_{1}\right) H_{i} p\left(t_{1}\right) .
\end{aligned}
$$

Combining this with (28) proves (19). Note that the proof so far used only first-order derivatives with respect to $s$.

To prove (20), fix an arbitrary $\alpha \in \mathcal{Q}^{k}$. Then by (21), $\sum_{i=1}^{k} \alpha_{i} H_{i} p\left(t_{1}\right)=0$ and this implies that $\sum_{i=1}^{k} \alpha_{i} G_{i} p(0)=$ 0 , so Prop. 1 yields $\tilde{H}(0, \alpha)\left(\left.\dot{\tilde{C}}\right|_{s=0}\right) v^{*}=0$, i.e.,

$$
\left(\left.\dot{\tilde{C}}\right|_{s=0}\right) v^{*}=0
$$

Substituting this in (30) yields

$$
\tilde{H}(0, \alpha)\left(\left.\ddot{\tilde{C}}\right|_{s=0}\right) v^{*}=\sum_{1 \leq i<j \leq k} \alpha_{i} \alpha_{j}\left[G_{i}, G_{j}\right] v^{*}
$$


and multiplying on the left by $q^{\prime}(0)$ gives

$$
\left(w^{*}\right)^{\prime}\left(\left.\ddot{\tilde{C}}\right|_{s=0}\right) v^{*}=q^{\prime}(0) \sum_{1 \leq i<j \leq k} \alpha_{i} \alpha_{j}\left[G_{i}, G_{j}\right] v^{*}
$$

On the other-hand, substituting (40) in (33) yields

$$
\left.\ddot{\tilde{\rho}}\right|_{s=0}=\left(w^{*}\right)^{\prime}\left(\left.\ddot{\tilde{C}}\right|_{s=0}\right) v^{*}
$$

so

$$
\begin{aligned}
z_{2}(\alpha) & =q^{\prime}(0) \sum_{1 \leq i<j \leq k} \alpha_{i} \alpha_{j}\left[G_{i}, G_{j}\right] p(0) \\
& =q^{\prime}\left(t_{1}\right) \sum_{1 \leq i<j \leq k} \alpha_{i} \alpha_{j}\left[H_{i}, H_{j}\right] p\left(t_{1}\right)
\end{aligned}
$$

and this proves (20). This completes the proof of Thm. 4.

\section{CONCLUSIONS}

The GUAS problem for switched systems is difficult because of the huge number of possible switching laws. This led to the variational approach that is based on trying to characterize a "most destabilizing" switching law.

For a PLSS, every possible switching law generates a positive transition matrix and the problem of finding the "most destabilizing" switching law is equivalent to finding the switching law that maximizes the spectral radius of the transition matrix.

In the relaxed version of a PLSS, i.e. a PBCS, this yields a well-defined optimal control problem, namely, for a fixed $T>0$, find a control $u$ that maximizes the spectral radius of the transition matrix at time T. A first-order necessary condition for optimality has been derived in [2]. In this paper, we derived a high-order necessary optimality condition for a singular control and for a bang-bang control. We demonstrated these conditions using simple examples. We are currently trying to use these high-order conditions to derive new theoretical results on the structure of the optimal control in specific problems. The main technical difficulty is that in general the Perron right and left eigenvectors $v^{*}$ and $w^{*}$ that appear in the optimality conditions are complicated functions of the entries of the transition matrix. 


\section{APPENDIX}

Proof of Thm. [1. Pick $t \geq 0$. Let $\Sigma^{t}:=\{C(t, u): u \in \mathcal{U}\}$, i.e., the set of all possible transition matrices at time $t$. The definition of $\mathcal{U}$ implies that $\Sigma^{t}$ is compact. Note that (7) yields

$$
\rho(A, B)=\limsup _{t \rightarrow \infty} \rho_{t}(A, B)=\limsup _{t \rightarrow \infty} \max _{M \in \Sigma^{t}}(\rho(M))^{1 / t}
$$

Pick a multiplicative matrix norm $\|\cdot\|: \mathbb{R}^{n \times n} \rightarrow \mathbb{R}_{+}$. By the Joint Spectral Radius Theorem (see, e.g., [10, Ch. 2]),

$$
\begin{aligned}
\rho(A, B) & =\limsup _{t \rightarrow \infty}\left\{\|M\|^{1 / t}: M \in \Sigma^{t}\right\} \\
& =\limsup _{t \rightarrow \infty}\left\{\|C(t, u)\|^{1 / t}: u \in \mathcal{U}\right\} .
\end{aligned}
$$

If $\rho(A, B)<1$ then

$$
\limsup _{t \rightarrow \infty}\{\|C(t, u)\|: u \in \mathcal{U}\}=0
$$

so $\lim _{t \rightarrow 0} C(t, u)=0$ for all $u \in \mathcal{U}$, and this implies GAS of the PBCS (4).

Now suppose that $\rho(A, B) \geqslant 1$. By [10, p. 22, Corollary 1.2], there exists a sequence $R_{1}, R_{2}, \ldots$ in $\Sigma^{t}$ such that

$$
\lim _{k \rightarrow \infty}\left\|R_{k} \ldots R_{1}\right\|^{1 / k} \geq 1
$$

In other words, there exists a sequence of controls $u_{i} \in \mathcal{U}$ such that

$$
\lim _{k \rightarrow \infty}\left\|C\left(t, u_{k}\right) \ldots C\left(t, u_{1}\right)\right\|^{1 / k} \geq 1
$$

Note that $C\left(t, u_{k}\right) \ldots C\left(t, u_{1}\right)$ is the transition matrix at time $k t$ for the control obtained by concatenating all the $u_{i}$ s. Thus, the PBCS is not GAS. This completes the proof.

\section{REFERENCES}

[1] G. Hochma and M. Margaliot, "Stability analysis of positive bilinear control systems: A variational approach," in IEEE 52nd Conference on Decision and Control (CDC 2013), Florence, Italy, 2013, pp. 1355-1359.

[2] L. Fainshil and M. Margaliot, "A maximum principle for positive bilinear control systems with applications to positive linear switched systems," SIAM J. Control Optim., vol. 50, pp. 2193-2215, 2012.

[3] D. Angeli, P. de Leenheer, and E. D. Sontag, "Chemical networks with inflows and outflows: A positive linear differential inclusions approach," Biotechnology Progress, vol. 25, pp. 632-642, 2009. 
[4] R. Shorten, F. Wirth, O. Mason, K. Wulff, and C. King, "Stability criteria for switched and hybrid systems," SIAM Review, vol. 49, pp. 545-592, 2007.

[5] M. S. Branicky, "Multiple Lyapunov functions and other analysis tools for switched and hybrid systems," IEEE Trans. Automat. Control, vol. 43, pp. 475-482, 1998.

[6] R. DeCarlo, M. Branicky, S. Pettersson, and B. Lennartson, "Perspectives and results on the stability and stabilizability of hybrid systems," Proc. IEEE, vol. 88, pp. 1069-1082, 2000.

[7] D. Liberzon and A. S. Morse, "Basic problems in stability and design of switched systems," IEEE Control Systems Magazine, vol. 19, pp. 59-70, 1999.

[8] D. Liberzon, Switching in Systems and Control. Birkhäuser, 2003.

[9] A. S. Morse, Ed., Control Using Logic-Based Switching. London: Springer, 1997.

[10] R. Jungers, The Joint Spectral Radius: Theory and Applications, ser. Lecture Notes in Control and Information Sciences. Springer, 2009, vol. 385 .

[11] M. Johansson, Piecewise Linear Control Systems, ser. Lecture Notes in Control and Information Sciences. Springer-Verlag, 2003, vol. 284.

[12] Z. Sun and S. S. Ge, Switched Linear Systems: Control and Design. Springer, 2005.

[13] Z. Sun and S. S. Ge, Stability Theory of Switched Dynamical Systems. Springer, 2011.

[14] L. Farina and S. Rinaldi, Positive Linear Systems: Theory and Applications. John Wiley, 2000.

[15] A. Berman and R. J. Plemmons, Nonnegative Matrices in the Mathematical Sciences. SIAM, 1987.

[16] M. A. Krasnoselskij, J. A. Lifshits, and A. V. Sobolev, Positive Linear Systems: the Method of Positive Operators. Heldermann-Verlag, 1989.

[17] O. Mason and R. Shorten, "A conjecture on the existence of common quadratic Lyapunov functions for positive linear systems," in Proc. 2003 American Control Conf., Denver, CO, 2003, pp. 4469-4470.

[18] L. Gurvits and A. Olshevsky, "On the NP-hardness of checking matrix polytope stability and continuous-time switching stability," IEEE Trans. Automat. Control, vol. 54, no. 2, pp. 337-341, 2009.

[19] L. Gurvits, R. Shorten, and O. Mason, "On the stability of switched positive linear systems," IEEE Trans. Automat. Control, vol. 52, pp. 1099-1103, 2007.

[20] L. Gurvits, "What is the finiteness conjecture for linear continuous time inclusions?" in Proc. 42nd IEEE Conf. on Decision and Control, Maui, HI, 2003, pp. 1165-1169.

[21] O. Mason, V. S. Bokharaie, and R. Shorten, "Stability and D-stability for switched positive systems," in Positive Systems, ser. Lecture Notes in Control and Information Sciences, R. Bru and S. Romero-Vivo, Eds. Springer-Verlag, 2009, vol. 389, pp. $101-109$.

[22] E. Fornasini and M. E. Valcher, "Linear copositive Lyapunov functions for continuous-time positive switched systems," IEEE Trans. Automat. Control, vol. 55, pp. 1933-1937, 2010.

[23] O. Ron, M. Margaliot, and M. S. Branicky, "Switching between linear consensus protocols: a variational approach," submitted. [Online]. Available: http://arxiv.org/abs/1407.2399

[24] M. Margaliot and M. S. Branicky, "Nice reachability for planar bilinear control systems with applications to planar linear switched systems," IEEE Trans. Automat. Control, vol. 54, pp. 1430-1435, 2009.

[25] L. Fainshil, M. Margaliot, and P. Chigansky, "On the stability of positive linear switched systems under arbitrary switching laws," IEEE Trans. Automat. Control, vol. 54, pp. 897-899, 2009.

[26] E. S. Pyatnitskii, "Absolute stability of nonstationary nonlinear systems," Automat. Remote Control, vol. 1, pp. 5-15, 1970. 
[27] E. S. Pyatnitskii, "Criterion for the absolute stability of second-order nonlinear controlled systems with one nonlinear nonstationary element," Automat. Remote Control, vol. 1, pp. 5-16, 1971.

[28] M. Margaliot, "Stability analysis of switched systems using variational principles: An introduction," Automatica, vol. 42, pp. 2059-2077, 2006.

[29] N. E. Barabanov, "Lyapunov exponent and joint spectral radius: Some known and new results," in Proc. 44th IEEE Conf. on Decision and Control, Seville, Spain, 2005, pp. 2332-2337.

[30] L. B. Rapoport, "Asymptotic stability and periodic motions of selector-linear differential inclusions," in Robust Control via Variable Structure and Lyapunov Techniques, ser. Lecture Notes in Control and Information Sciences, F. Garofalo and L. Glielmo, Eds. Springer, 1996, vol. 217, pp. 269-285.

[31] M. Balde and U. Boscain, "Stability of planar switched systems: the nondiagonalizable case," Communications Pure Applied Analysis, vol. 7, pp. 1-21, 2008.

[32] M. Balde, U. Boscain, and P. Mason, “A note on stability conditions for planar switched systems,” Int. J. Control, vol. 82, pp. 1882-1888, 2009.

[33] T. Monovich and M. Margaliot, "Analysis of discrete-time linear switched systems: A variational approach," SIAM J. Control Optim., vol. 49, pp. 808-829, 2011.

[34] T. Monovich and M. Margaliot, "A second-order maximum principle for discrete-time bilinear control systems with applications to discrete-time linear switched systems," Automatica, vol. 47, pp. 1489-1495, 2011.

[35] M. Margaliot and M. S. Branicky, "Stability analysis of positive linear switched systems: a variational approach," in Proc. 6th IFAC Symposium on Robust Control Design (ROCOND09), Haifa, Israel, 2009.

[36] A. F. Filippov, "On certain questions in the theory of optimal control," SIAM J. Control Optim., vol. 1, pp. 76-84, 1962.

[37] R. A. Horn and C. R. Johnson, Matrix Analysis, 2nd ed. Cambridge University Press, 2013.

[38] E. Deutsch and M. Neumann, "Derivatives of the Perron root at an essentially nonnegative matrix and the group inverse of an M-matrix," J. Math. Anal. Appl., vol. 102, no. 1, pp. 1-29, 1984.

[39] A. A. Agrachev and M. Sigalotti, “On the local structure of optimal trajectories in R ${ }^{3}$," SIAM J. Control Optim., vol. 42, pp. 513-531, 2003.

[40] H. Hermes, "Lie algebras of vector fields and local approximation of attainable sets," SIAM J. Control Optim., vol. 16, pp. 715-727, 1978.

[41] A. J. Krener, "The high order maximal principle and its applications to singular extremals," SIAM J. Control Optim., vol. 15, pp. 256-293, 1977.

[42] I. Ratmansky and M. Margaliot, "A simplification of the Agrachev-Gamkrelidze second-order variation for bang-bang controls," Systems Control Lett., vol. 59, pp. 25-32, 2010.

[43] Y. Sharon and M. Margaliot, “Third-order nilpotency, finite switchings and asymptotic stability,” J. Diff. Eqns., vol. 233, pp. 136-150, 2007. 